\title{
Peritonitis from Anaerobic Gram-positive Cocci Likely Due to Translocation of Bacteria from Gut in a Patient Undergoing Peritoneal Dialysis
}

\author{
Sreedhar Adapa ${ }^{1}$, Srikanth Naramala ${ }^{2}$, Daniel Boken ${ }^{3}$, Alan Moreno ${ }^{1}$, Venu Madhav Konala ${ }^{4}$ \\ 1. Internal Medicine/Nephrology, Kaweah Delta Medical Center, Visalia, USA 2. Rheumatology, Adventist Medical \\ Center, Hanford, USA 3. Internal Medicine/Infectious Disease, Kaweah Delta Medical Center, Visalia, USA 4. Internal \\ Medicine/Hematology and Oncology, Ashland Bellefonte Cancer Center, Ashland, USA
}

Corresponding author: Sreedhar Adapa, sreedharadapa@gmail.com

\begin{abstract}
Peritonitis is a severe complication encountered in patients undergoing peritoneal dialysis, often causing high morbidity and mortality. High vigilance is required from healthcare providers involved in the management of these patients to prevent this complication. There has been recognition of peritonitis caused by unusual organisms because of improved microbiological detection techniques. Gram-positive organisms are the frequent cause of peritonitis compared to Gram-negative organisms. We report a rare case of peritonitis caused by Enterococcus avium. A 60-year-old male on peritoneal dialysis presented with palpitations, abdominal pain, diarrhea, and cloudy effluent. The peritoneal fluid analysis was consistent with peritonitis, and peritoneal fluid culture grew E. avium. The patient was treated with linezolid after failing to respond to vancomycin. The peritoneal dialysis catheter was removed, and the dialysis modality has been switched to hemodialysis because of refractory peritonitis.
\end{abstract}

Received 10/07/2019

Review began 10/18/2019 Review ended 11/01/2019 Published 11/03/2019

\section{() Copyright 2019}

Adapa et al. This is an open access article distributed under the terms of the Creative Commons Attribution License CC-BY 3.0., which permits unrestricted use, distribution, and reproduction in any medium, provided the original author and source are credited.
Categories: Internal Medicine, Infectious Disease, Nephrology

Keywords: enterococcus avium, peritonitis, enterococcus

\section{Introduction}

Enterococcus avium is Gram-positive, catalase-negative cocci, and facultative anaerobe [1]. It is a normal commensal of the gastrointestinal and genitourinary tract in humans and animals. The organism was isolated initially from human feces in 1955 [2]. Peritonitis is a significant complication encountered in patients undergoing peritoneal dialysis, which causes significant morbidity and mortality [3]. The incidence of peritonitis from rare organisms is increasing because of improved microbiological identification techniques. We report a case of E. avium associated peritonitis.

\section{Case Presentation}

A 60-year-old male was admitted with a chief complaint of palpitations, abdominal pain, and diarrhea. Palpitations were sudden in onset and woke up the patient from sleep and were persistent, which prompted him to seek medical attention. The patient was having diarrhea and abdominal pain for two days before the presentation. Past medical history was significant for polycystic kidney disease resulting in end-stage renal disease, bilateral nephrectomies with living unrelated kidney transplant that failed after 13 years, and he was on peritoneal dialysis for two years. Other history details include hypertension, diabetes, hyperlipidemia, and coronary artery disease with four-vessel coronary artery bypass grafting. Home medications include aspirin 81 milligrams (mg) daily, ticagrelor $90 \mathrm{mg}$ twice a day, calcitriol 0.25 micrograms daily, carvedilol $6.25 \mathrm{mg}$ twice a day, olmesartan $40 \mathrm{mg}$ daily, vitamin d2 50,000 units weekly, and insulin sliding scale.

The vital signs in the ER were a temperature of 36.5 centigrade, pulse rate of 80 beats per minute (bpm), respiratory rate of 18 breaths per minute, and blood pressure of 123/76 mmHg. Physical examination revealed that the patient was in sinus rhythm, with no rubs or gallops or murmurs. Abdominal examination revealed a distended, tender abdomen with a peritoneal dialysis catheter in the left lower quadrant. The rest of the physical examination was unremarkable.

Investigations revealed electrocardiogram with $80 \mathrm{bpm}$ in sinus rhythm, premature complexes, and nonspecific conduction delay as shown in Figure 1. Laboratory data showed hemoglobin $7.8 \mathrm{~g} / \mathrm{dL}$, white blood cell (WBC) count $7220 \mathrm{~mm}^{3}$, platelet count $150,000 \mathrm{~mm}^{3}$, sodium $131 \mathrm{mmol} / \mathrm{L}$, potassium $4.3 \mathrm{mmol} / \mathrm{L}$, bicarbonate $20 \mathrm{mmol} / \mathrm{L}$, blood urea nitrogen $76 \mathrm{mg} / \mathrm{dL}$, creatinine $12.92 \mathrm{mg} / \mathrm{dL}$, albumin $2.8 \mathrm{~g} / \mathrm{dL}$, and troponin I $15.01 \mathrm{ng} / \mathrm{mL}$. The peritoneal fluid effluent revealed peritoneal fluid WBC 14,309 cells/uL, with $89 \%$ predominant neutrophils. Peritoneal fluid Gram stain revealed $>100 \mathrm{WBC}$, and no organisms were seen. The patient was started on treatment for peritonitis with empiric intraperitoneal vancomycin and ceftazidime. The patient underwent coronary intervention because of non-ST elevation myocardial infarction (NSTEMI) and had successful angioplasty of in-stent restenosis of ramus intermedius. The 


\section{Cureus}

peritoneal fluid WBC count started to get better with 11,715 cells/uL on day 2,4502 cells/uL on day 3,1574 cells/uL on day four and got worse to 10,097 cells/uL on day 5. Peritoneal fluid culture grew $E$. avium in both aerobic and anaerobic bottles. Species identification was made by VITEK 2. Sensitivities were done using a broth microdilution technique. The sensitivities of E. avium to antibiotic are listed in Table 1 .

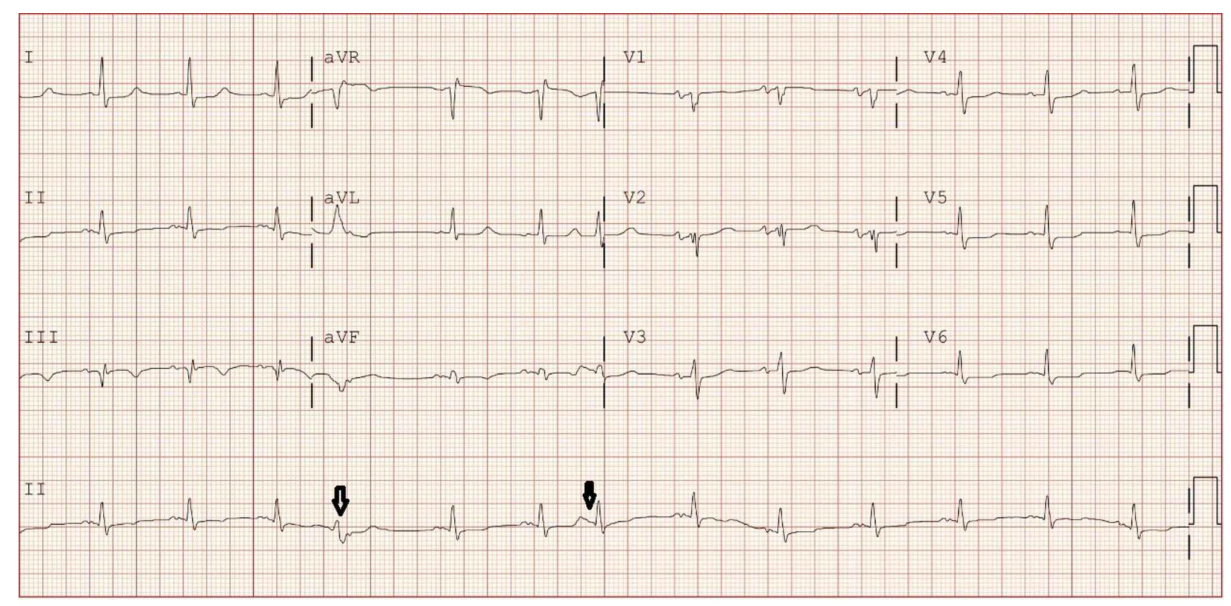

FIGURE 1: Electrocardiogram - sinus rhythm at 80 beats per minute and premature complexes shown in black arrows.

\begin{tabular}{|c|c|c|}
\hline Antibiotic & MIC (minimum inhibitory concentration) & Antibiotic sensitivity \\
\hline Ampicillin & $<=4 \mathrm{mcg} / \mathrm{mL}$ & $S$ \\
\hline Daptomycin & $0.5 \mathrm{mcg} / \mathrm{mL}$ & $S$ \\
\hline Linezolid & $1 \mathrm{mcg} / \mathrm{mL}$ & $\mathrm{s}$ \\
\hline Vancomycin & $<=0.5 \mathrm{mcg} / \mathrm{mL}$ & $S$ \\
\hline
\end{tabular}

TABLE 1: Antibiotic sensitivities of Enterococcus avium.

S - sensitive

The patient was initially treated with vancomycin and was changed to linezolid because of worsening peritoneal fluid cell count before the antibiotic sensitivities were available. The peritoneal dialysis catheter was removed, and the dialysis modality has been switched to hemodialysis because of refractory peritonitis. The patient was discharged on linezolid and completed the antibiotic course for a total of 14 days. He was followed up in the clinic after completion of antibiotics and was doing well without any signs and symptoms of recurrent infection. He was continued on hemodialysis three times a week as an outpatient for end-stage renal disease.

\section{Discussion}

Enterococcus species are normal commensals of human gut flora. They cause urinary tract infections, intraabdominal infections, bacteremia, and nosocomial infections that are antibiotic-resistant frequently [4]. Ninety percent of infections caused by Enterococcus species are attributed to E. faecalis and E. faecium [5]. Other clinically important species in Enterococci are E. avium, E. flavescens, E. casseliflavus, and E. gallinarium, which accounts for less than five percent of clinically significant infections [5].

Enterococcus avium was formerly placed in group $\mathrm{Q}$ streptococcus as it has group $\mathrm{Q}$ antigen [6]. Streptococcus avium derives its name as they were frequently isolated from chicken feces [6] and subsequently were isolated from feces of all mammals. The bacterium was later transferred to genus Enterococcus in 1980 as the biochemical features were similar to Enterococcus species [7].

Enterococcus avium has low virulence [8] and is an opportunistic pathogen in an immunocompromised host. The bacterium colonizes in the gastrointestinal and genitourinary tract, and the mode of transmission could 
be from contamination of devices or translocation from the colonized body sites. Most of the case reports or series that had been reported in the literature have severe gastrointestinal diseases. They are often associated with other gastrointestinal organisms, and infections tend to be polymicrobial [9]. E. avium has been isolated from the blood and bile of immunocompetent host with acute cholecystitis before [10]. E. avium has been reported to cause bacteremia, meningitis, endocarditis, and intra-abdominal infections. $E$. avium rarely causes peritonitis, but only a few cases were reported so far as per the review of the literature [11-12].

Enterococcus avium is readily identified by the routine blood and body fluid culture. Commonly used media to identify Enterococcus species include bile esculin agar and 6.5\% salt broth [12]. Species identification techniques include VITEK 2 automated system, matrix-assisted laser desorption/ionization-time of flight (MALDI-TOF), polymerase chain reaction (PCR) for specific genes, 16S rRNA sequencing, and proprietary multiplexed nucleic acid amplification [13-16].

Enterococcus avium is susceptible to most of the antibiotics, which differentiates, from $E$. faecalis and $E$. faecium that tend to be resistant to them [9-10]. However, our patient was initially treated with vancomycin and responded with decreased peritoneal fluid white cell count and then got worse; hence, vancomycin was switched to linezolid before the sensitivities were available. Prompt identification and timely initiation of antibiotics and appropriate intervention will prevent mortality associated with this organism.

\section{Conclusions}

Enterococcus avium is an organism with low virulence which causes opportunistic infections. Peritonitis from E. avium in patients receiving peritoneal dialysis is rare and recent diagnostic methods aid in the identification of this organism. The bacterium is usually sensitive to common antibiotics.

\section{Additional Information \\ Disclosures}

Human subjects: Consent was obtained by all participants in this study. Conflicts of interest: In compliance with the ICMJE uniform disclosure form, all authors declare the following: Payment/services info: All authors have declared that no financial support was received from any organization for the submitted work. Financial relationships: All authors have declared that they have no financial relationships at present or within the previous three years with any organizations that might have an interest in the submitted work. Other relationships: All authors have declared that there are no other relationships or activities that could appear to have influenced the submitted work.

\section{References}

1. Jones S, England R, Evans M, Soo SS, Venkatesan P: Microbiologically confirmed meningoencephalitis due to Enterococcus avium: a first report. J Infect. 2007, 54:129-131. 10.1016/j.jinf.2006.08.012

2. Patel R, Keating MR, Cockerill III FR, Steckelberg JM: Bacteremia due to Enterococcus avium. Clin Infect Dis. 1993, 17:1006-1111.10.1093/clinids/17.6.1006

3. Adapa S, Gumaste P, Konala VM, Agrawal N, Garcha AS, Dhingra H: Peritonitis due to Moraxella osloensis: an emerging pathogen. Case Rep Nephrol. 2018 , 2018:10.1155/2018/4968371

4. Caballero-Granado FJ, Becerril B, Cuberos L, Bernabeu M, Cisneros JM, Pachon J: Attributable mortality rate and duration of hospital stay associated with enterococcal bacteremia. Clin Infect Dis. 2001, 15:587-594. $10.1086 / 318717$

5. Huycke MM, Sahm DF, Gilmore MS: Multiple-drug resistant enterococci: the nature of the problem and an agenda for the future. Emerg Infect Dis. 1998, 4:239.

6. Nowlan SS, Deibel RH: Group Q streptococci. I. Ecology, serology, physiology, and relationship to established enterococci. J Bacteriol. 1967, 94:291-296.

7. Collins MD, Jones D, Farrow JA, Kilpper-Balz R, Schleifer KH: Enterococcus avium nom. rev., comb. nov.; E. casseliflavus nom. rev., comb. nov.; E. durans nom. rev., comb. nov.; E. gallinarum comb. nov.; and E. malodoratus sp. nov.. Int J Syst Evolut Microbiol. 1984, 34:220-223. 10.1099/00207713-34-2-220

8. Fisher K, Phillips C: The ecology, epidemiology and virulence of Enterococcus . Microbiology. 2009, 155:1749-1757. 10.1099/mic.0.026385-0

9. Na S, Park HJ, Park KH, et al.: Enterococcus avium bacteremia: a 12-year clinical experience with 53 patients. Eur J Clin Microbiol Infect Dis. 2012, 1:303-310. 10.1007/s10096-011-1311-1

10. Verhaegen J, Pattyn P, Hinnekens P, Colaert J: Isolation of Enterococcus avium from bile and blood in a patient with acute cholecystitis. J Infect. 1997, 35:77-78. 10.1016/S0163-4453(97)91097-5

11. Chao CT, Yang SY, Huang JW: Peritoneal dialysis peritonitis caused by Enterococcus avium. Perit Dial Int. 2013, 33:335-336. 10.3747/pdi.2012.00166

12. Ugur AR, Findik D, Dagi HT, Tuncer I, Peru H: Enterococcus avium peritonitis in a child on continuous ambulatory peritoneal dialysis. Perit Dial Int. 2014, 34:127-128. 10.3747/pdi.2012.00241

13. Depardieu F, Perichon B, Courvalin P: Detection of the van alphabet and identification of Enterococci and Staphylococci at the species level by multiplex PCR. J Clin Microbiol. 2004, 42:5857.

14. Martinez RM, Bauerle ER, Fang FC, Butler-Wu SM: Evaluation of three rapid diagnostic methods for direct identification of microorganisms in positive blood cultures. J Clin Microbiol. 2014, 52:2521.

10.1128/JCM.00529-14

15. Deck MK, Anderson ES, Buckner RJ, et al.: Rapid detection of Enterococcus spp. direct from blood culture 


\section{Cureus}

bottles using Enterococcus QuickFISH method: a multicenter investigation. Diagn Microbiol Infect Dis

2014, 78:338. 10.1016/j.diagmicrobio.2013.12.004

16. Fang H, Ohlsson AK, Ullberg M, Ozenci V: Evaluation of species-specific PCR, Bruker MS, VITEK MS and the

VITEK 2 system for the identification of clinical Enterococcus isolates. Eur J Clin Microbiol Infect Dis. 2012, 31:3073. 10.1007/s10096-012-1667-x 